\title{
Specimen-Specific Method for Quantifying Glenohumeral Joint Kinematics
}

\author{
YeOn Soo LeE ${ }^{1,2,3}$ and Thay Q. LeE ${ }^{1,2}$ \\ ${ }^{1}$ Orthopaedic Biomechanics Laboratory, VA Long Beach Healthcare System (09/151), 5901 East 7th Street, Long Beach, \\ CA 90822, USA; ${ }^{2}$ Department of Orthopaedic Surgery, University of California, Irvine, CA, USA; and ${ }^{3}$ Department of \\ Biomedical Engineering, College of Medical Science, Catholic University of Daegu, Gyungbook, Republic of Korea
}

(Received 4 November 2009; accepted 11 May 2010; published online 25 May 2010)

Associate Editor Eric M. Darling oversaw the review of this article.

\begin{abstract}
The existing glenohumeral joint kinematic protocols are highly effective for studying in vivo shoulder kinematics but are not anatomically specific enough to address the asymmetric changes in glenohumeral joint kinematics and do not provide clear anatomic definitions for landmarks and directions. Therefore, the objective of this study was to develop an anatomically relevant and specimenspecific three-dimensional glenohumeral joint kinematic method as a new standard definition protocol for the glenohumeral coordinate systems (CSs). The in situ kinematic data of the intra-capsular glenoid-based CS of the glenohumeral joint were mathematically determined from the kinematic data of the extra-capsular CSs measured with an intact capsule. To minimize irreproducibility arising from discrepancy in initial specimen condition and error in determining CSs, several techniques were employed to determine anatomical landmarks and directions. To examine and demonstrate the details of this method, six fresh frozen cadaveric shoulders were used with a custom shoulder testing system. The accuracy and repeatability in the humeral head center (HHC) measurement were 0.44 and $0.41 \mathrm{~mm}$, respectively. The inter-observer reliability for the location of the glenoid CS origin and HHC were 0.37 and $0.30 \mathrm{~mm}$, respectively. The smaller anteroposterior (AP) depth of the glenoid with respect to the superoinferior $(\mathrm{SI})$ depth $(27.3 \pm 16.5 \%)$ was significantly correlated to the larger AP/SI translation ratio of the humeral head apex $(191.4 \pm 43.8 \%, R=0.90$, $p=0.02$ ). This study provides a glenohumeral kinematic protocol that enables the assessment of asymmetric glenohumeral kinematics determined by a precise and reproducible method using anatomic landmarks.
\end{abstract}

Keywords-Shoulder, Kinematics, Glenohumeral Joint, Coordinate system, Landmarks.

Address correspondence to Thay Q. Lee, Orthopaedic Biomechanics Laboratory, VA Long Beach Healthcare System (09/151), 5901 East 7th Street, Long Beach, CA 90822, USA. Electronic mail: biomechanics.yslee@gmail.com, tqlee@med.va.gov, tqlee@uci.edu

\section{NOMENCLATURE}

\begin{tabular}{|c|c|}
\hline $\mathrm{A}, \mathrm{P}, \mathrm{S}, \mathrm{I}$ & $\begin{array}{l}\text { Anterior, posterior, superior, and } \\
\text { inferior points of the bony } \\
\text { glenoid rim }\end{array}$ \\
\hline$\vec{A}, \vec{P}, \vec{S}, \vec{I}$ & $\begin{array}{l}\text { Vector expression of } \mathrm{A}, \mathrm{P}, \mathrm{S}, \mathrm{I} \\
\text { respectively }\end{array}$ \\
\hline AP & $\begin{array}{l}\text { Direction of anterior }(+) \text { to } \\
\text { posterior }(-)\end{array}$ \\
\hline CACS & $\begin{array}{l}\text { Coracoacromial coordinate } \\
\text { system }(\mathrm{CS} 1)\end{array}$ \\
\hline $\begin{array}{l}\mathrm{CS} 1, \mathrm{CS} 2, \mathrm{CS} 3 \\
\mathrm{CS} 4, \mathrm{CS} 5\end{array}$ & $\begin{array}{l}\text { Abbreviations of coordinate } \\
\text { systems } 1,2,3,4,5\end{array}$ \\
\hline GCS & $\begin{array}{l}\text { Glenoid coordinate system (CS3) } \\
\text { whose origin is } \vec{O}_{3}\end{array}$ \\
\hline HHA & Humeral head apex $\left(H_{\mathrm{A}}\right.$ or $\left.O_{4}\right)$ \\
\hline $\mathrm{HHC}$ & Humeral head center $\left(H_{\mathrm{C}}\right)$ \\
\hline HHCS & $\begin{array}{l}\text { Humeral head coordinate system } \\
\text { (CS4) }\end{array}$ \\
\hline HSA & Humeral shaft axis \\
\hline HSC & $\begin{array}{l}\text { A proximal point along the } \\
\text { humeral shaft axis }\end{array}$ \\
\hline HSCS & $\begin{array}{l}\text { Humeral shaft coordinate system } \\
\text { (CS5) }\end{array}$ \\
\hline LM & $\begin{array}{l}\text { Direction of lateral }(+) \text { to } \\
\text { medial }(-)\end{array}$ \\
\hline$\vec{O}_{1}, \vec{O}_{2}, \vec{O}_{3}, \vec{O}_{4}, \vec{O}_{5}$ & $\begin{array}{l}\text { Origins of } \mathrm{CS} 1, \mathrm{CS} 2, \mathrm{CS} 3, \mathrm{CS} 4 \text {, } \\
\mathrm{CS} 5 \text {, respectively }\end{array}$ \\
\hline$\vec{O}_{3}^{\prime}$ & $\begin{array}{l}\text { origin of pre-GCS determined by } \\
\text { the points } \mathrm{A}, \mathrm{P}, \mathrm{S}, \mathrm{I}\end{array}$ \\
\hline PHCS & $\begin{array}{l}\text { Proximal humerus coordinate } \\
\text { system (CS2) }\end{array}$ \\
\hline$Q$ & The summation of all $Q_{i}$ \\
\hline$Q_{i}$ & The area of a triangle $\Delta\left(\overrightarrow{\bar{p}}, \vec{p}_{i}, \vec{p}_{i+1}\right)$ \\
\hline SI & $\begin{array}{l}\text { Direction of superior }(+) \text { to } \\
\text { inferior }(-)\end{array}$ \\
\hline
\end{tabular}




\begin{tabular}{|c|c|}
\hline$a 1, a 2, a 3$ & $\begin{array}{l}\text { Digitized points } 1,2,3 \text { on the } \\
\text { coracoacromial complex }\end{array}$ \\
\hline$c 1, c 2$ & $\begin{array}{l}\text { Centers of the humeral shaft } \\
\text { circumference } 1 \text { and } 2\end{array}$ \\
\hline$\vec{i}, \vec{j}, \vec{k}$ & $\begin{array}{l}U \text { Unit direction vector of } X \text {-axis, } \\
Y \text {-axis, and } Z \text {-axis, respectively }\end{array}$ \\
\hline$\vec{g}_{h}$ & $\begin{array}{l}\text { Point among } \vec{g}_{j} \text { that has the least } \\
\text { distant from } \vec{O}_{3}^{\prime}\end{array}$ \\
\hline$\vec{g}_{j}$ & $\begin{array}{l}\text { Vector expression of a } j \text { th } \\
\text { digitized point on the glenoid } \\
\text { articular surface }\end{array}$ \\
\hline$h 1, h 2, h 3$ & $\begin{array}{l}\text { Digitized points } 1,2,3 \text { on the } \\
\text { proximal humerus }\end{array}$ \\
\hline maxER & Maximum external rotation \\
\hline $\operatorname{maxIR}$ & Maximum internal rotation \\
\hline$\vec{n}$ & Average normal vector of all $\vec{n}_{i}$ \\
\hline$\vec{n}_{i}$ & $\begin{array}{l}\text { Normal vector to the plane of } \\
\Delta\left(\vec{p}, \vec{p}_{i}, \vec{p}_{i+1}\right)\end{array}$ \\
\hline $\overrightarrow{\bar{p}}$ & $\begin{array}{l}\text { Vector expression of the average } \\
\text { position of all } \vec{p}_{i}\end{array}$ \\
\hline$\vec{p}_{\mathrm{HHC}}$ & $\begin{array}{l}\text { Vector expression of the humeral } \\
\text { head center }\end{array}$ \\
\hline$\vec{p}_{i}$ & $\begin{array}{l}\text { Vector expression of the digitized } \\
\text { points along the anatomical } \\
\text { humeral neck line }\end{array}$ \\
\hline pre-GCS & $\begin{array}{l}\text { The preliminary GCS whose } \\
\text { origin is } \vec{O}_{3}^{\prime} \text { and whose axes are } \\
X_{3}^{\prime}, Y_{3}^{\prime}, Z_{3}^{\prime}\end{array}$ \\
\hline$s_{i}$ & Area fraction ratio \\
\hline$\alpha_{i}$ & $\begin{array}{l}\text { Digitized points on the convex } \\
\text { articulating surface of the } \\
\text { humeral head }\end{array}$ \\
\hline$\Delta h$ & $\begin{array}{l}\text { The distance between HHA and } \\
\text { HHC }\end{array}$ \\
\hline$\Delta\left(\overrightarrow{\bar{p}}, \vec{p}_{i}, \vec{p}_{i+1}\right)$ & $\begin{array}{l}\text { A triangle which is composed of } \\
\vec{p}, \vec{p}_{i}, \vec{p}_{i+1}\end{array}$ \\
\hline$\Delta t$ & Translation of $\vec{O}_{3}^{\prime}$ to $\vec{O}_{3}$ \\
\hline
\end{tabular}

\section{INTRODUCTION}

Anatomically precise three-dimensional (3D) glenohumeral joint kinematics are essential for understanding detailed glenohumeral joint function. Glenohumeral articulation is a 3D movement between an ellipsoidal humeral head and a concave glenoid. In addition, the articulation is an asymmetric surface gliding mechanism. ${ }^{1,2,15}$ The current biomechanical methods for assessing glenohumeral joint kinematics focus on simple motions such as joint translation and range of motion without detailed 3D asymmetric anatomy consideration. Most current techniques are highly effective for studying in vivo shoulder kinematics but are not capable of addressing the complex asymmetric changes in the kinematics of the glenohumeral joint.

The current International Society of Biomechanics (ISB) recommendation provides a definition for the scapula coordinate system (CS) including the acromion and the coracoid rather than a definition for the glenoid CS. ${ }^{24}$ This is highly effective for studying in vivo shoulder kinematics; however, it is not possible to use the current ISB recommendation to accurately quantify anatomically relevant glenohumeral joint kinematics because it does not define a specific glenoid CS that is crucial for assessing the humeral movement with respect to the glenoid. The ISB recommendation is to encourage communication among researchers, clinicians, and all other interested parties. ${ }^{24}$ In the ISB recommendation, the glenohumeral kinematic center is proposed to be used as a common landmark for the scapula and humerus CSs 'since the glenohumeral joint resembles a ball-and-socket joint. ${ }^{24}$ However, due to the complex asymmetric articulation between the humerus and the glenoid the glenohumeral joint does not behave as a simple ball-and-socket joint. Also, the kinematic rotation center is only able to be determined when a series of humeral rotational motion data are provided and as stated in the ISB recommendation the determination of this center can only be a 'rough estimate.' Due to the lack of clear landmark determination protocol, the current ISB recommendation is not able to reveal the influence of the asymmetric anatomy on the glenohumeral joint. ${ }^{8,27}$ The objective of this study was to devise an anatomically relevant specimen specific 3D glenohumeral joint kinematic method for quantifying asymmetric glenohumeral joint kinematics. ${ }^{8,26-28}$ This study proposes an anatomically relevant specimen-specific 3D glenohumeral joint kinematic method as a new standard definition protocol for the glenohumeral CSs.

\section{METHODS}

\section{Experimental Design}

The experiment for quantifying anatomically based glenohumeral joint kinematics can be divided into three processes: (1) in situ movement data collection of extra-capsular CSs, (2) construction of intra-articular CSs and an extra-capsular humeral shaft coordinate system (HSCS), and (3) calculation of glenohumeral kinematics (Fig. 1). All data and CSs were referred to a local CS. The local CS was defined at the start of the experiment and was used as a reference system throughout the experiment.

For in situ kinematic data collection, extra-capsular landmarks were assigned on the surfaces of the coracoacromial complex and the proximal humerus so that 


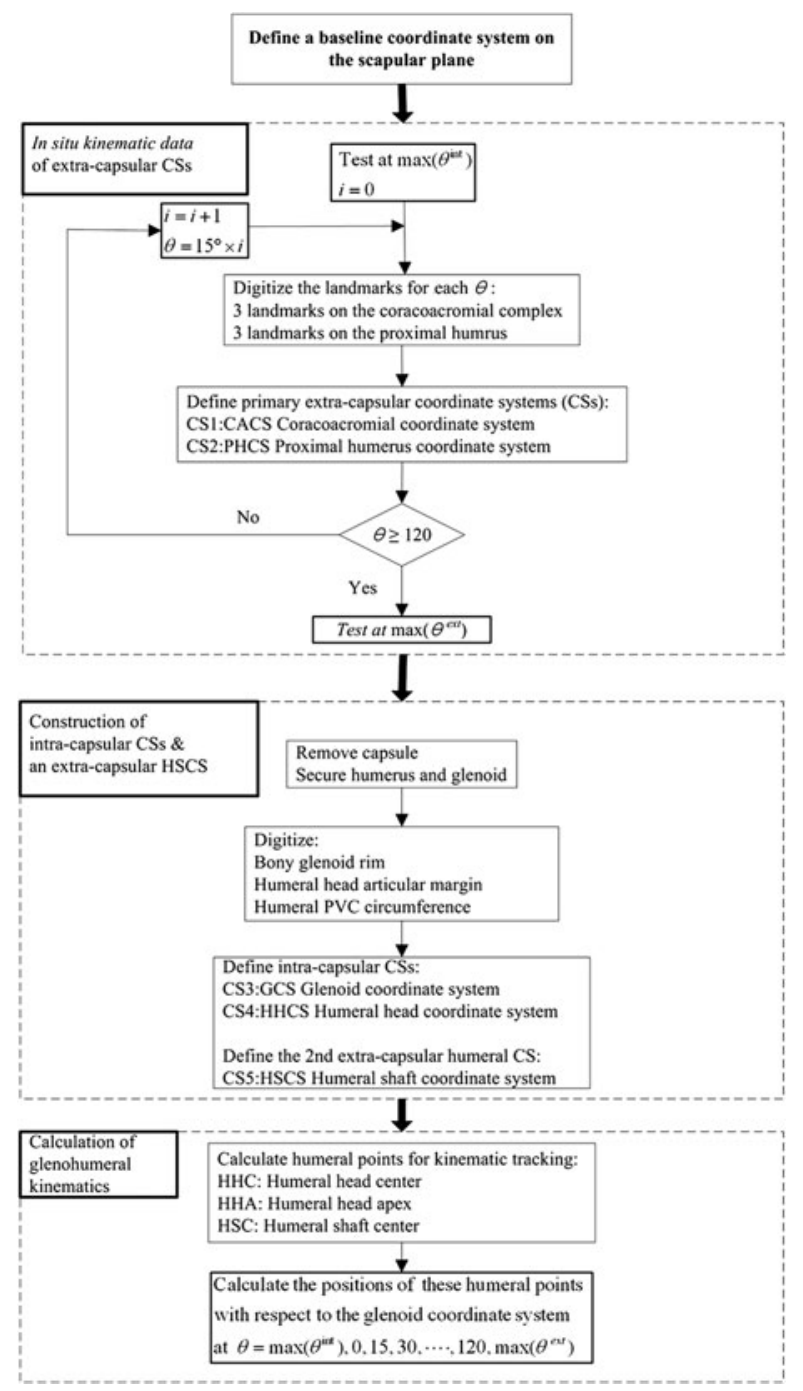

FIGURE 1. A flowchart showing the experimental design.

they could be digitized without violating the glenohumeral capsule. For digitizing, six small crosshead screws were inserted, three on the scapula and three on the humerus. Using these anatomic landmarks, two extra-articular CSs were defined: the coracoacromial coordinate system (CS1:CACS) and the proximal humerus coordinate system (CS2:PHCS).

Once the in situ kinematic tests were completed, the joint capsule was removed and the anatomic data for the glenoid and the humerus were collected. This is a critical step to obtain morphological data for the calculation of anatomically based glenohumeral joint kinematics because when testing a shoulder specimen with an intact capsule, the intra-capsular anatomical landmarks cannot be digitized directly. Based on these anatomic data, two intra-capsular CSs and one extracapsular CS were defined: the glenoid coordinate system (CS3:GCS), the humeral head coordinate system
(CS4:HHCS), and the humeral shaft coordinate system (CS5:HSCS).

The glenohumeral kinematic path was calculated using spatial relationships among the intra-capsular and extra-capsular CSs. Because these bony structures are rigid, there is a constant spatial relationship between the CSs. The kinematic data of each humeral anatomic landmark were calculated at each degree of humeral rotation using this constant spatial relationship.

\section{Specimens}

Six macroscopically intact fresh frozen cadaveric shoulders from six donors (four rights, two lefts, five females, one male, average age: 78.3 years, range 49 97) were used. Soft tissues except the glenohumeral joint capsule were carefully dissected from the scapula and the humerus.

The scapula was fixed and potted in a rectangular box with plaster of Paris (DAP Products Inc., Baltimore, MD, USA). To position the scapula reproducibly a method was devised to align the glenoid with the rectangular box along the superoinferior (SI) and anteroposterior (AP) directions using Kirschner wires (DePuy Orthopaedics Inc., Warsaw, IN, USA). The SI axis was defined from 12 to 6 o'clock on the glenoid and the AP axis was defined from 3 to 9 o'clock for right glenoids and 9 to 3 o'clock for left glenoids.

The humerus was fixed in a $45-\mathrm{mm}$ diameter polyvinyl chloride (PVC) cylinder using screws and plaster of Paris. The humeral shaft was transected $200 \mathrm{~mm}$ distal to the deltoid tuberosity and the axis of the distal humerus was determined using an intramedullary rod based on the distal $100 \mathrm{~mm}$ of humeral shaft. The longitudinal axial center line of the PVC pipe was matched to the centerline of the humerus. ${ }^{13,17,19,26-28}$

\section{Shoulder Testing System}

Biomechanical testing of each shoulder was performed at $60^{\circ}$ of glenohumeral abduction in the scapular plane using a custom shoulder testing system (Fig. 2). The system permits six degree-of-freedom for positioning of the glenohumeral joint. ${ }^{7,11,12,18,20,28}$ The humeral rotational range of motion was measured using a physical goniometer placed at the distal end of the humerus. This goniometer was inscribed with $1^{\circ}$ increments, making the precision of the measurement $0.5^{\circ}$. The glenohumeral joint kinematics was determined by digitizing anatomic landmarks with a 3D digitizing system, the MicroScribe 3DLX (Revware Inc., Raleigh, NC, USA). The accuracy and repeatability of the MicroScribe 3DLX are 0.3 and $0.2 \mathrm{~mm}$, respectively. ${ }^{17}$ 


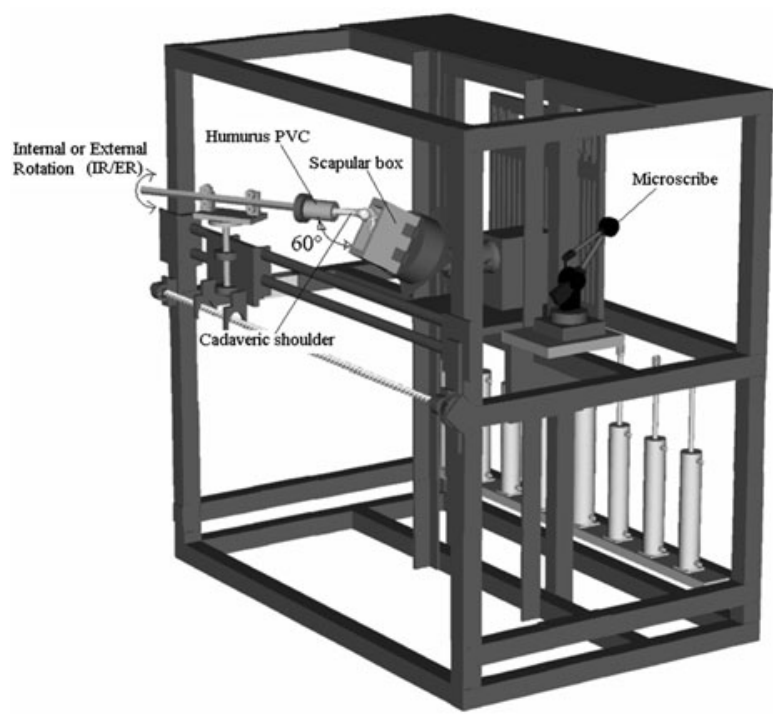

FIGURE 2. Custom shoulder testing system.

\section{External and Internal Humeral Rotations}

The axial rotation of the humerus was measured by defining $90^{\circ}$ of external rotation as the position where the bicipital groove was aligned with the anterior edge of the acromion. ${ }^{10,21}$ The neutral humeral rotation position $\left(0^{\circ}\right.$ of rotation) was then determined as $90^{\circ}$ of internal rotation from the established $90^{\circ}$ of external rotation position. ${ }^{13}$ Twenty-two Newtons of glenohumeral joint compressive force was applied perpendicular to the glenoid using a cable attached to a rotational bearing at the distal end of the humerus. The $22 \mathrm{~N}$ of compression has been shown to provide enough compressive force, under no muscle loading, to keep the joint located..$^{8,19,26,27}$ The maximum internal and external rotation positions were determined with $2.2 \mathrm{Nm}$ of torque. ${ }^{8,19,26,27}$ The positions of the humerus were recorded at maximum internal rotation and from neutral to $120^{\circ}$ external rotation in $15^{\circ}$ increments, followed by maximum external rotation. All specimens were kept moist throughout the experiment with normal saline.

\section{Anatomically Based Glenohumeral Joint Kinematics}

All kinematic data were referred to a baseline local CS. The baseline local CS was defined on the scapular mounting plate that represented the glenoid surface plane at the beginning of the experiment and this CS was used as a reference CS throughout the experiment.

Five additional local CSs were then defined. First, two primary extra-capsular CSs were defined in the proximal humerus and the coracoacromial complex, i.e., the coracoacromial CS and the proximal humerus CS. Second, when kinematic data collection of the

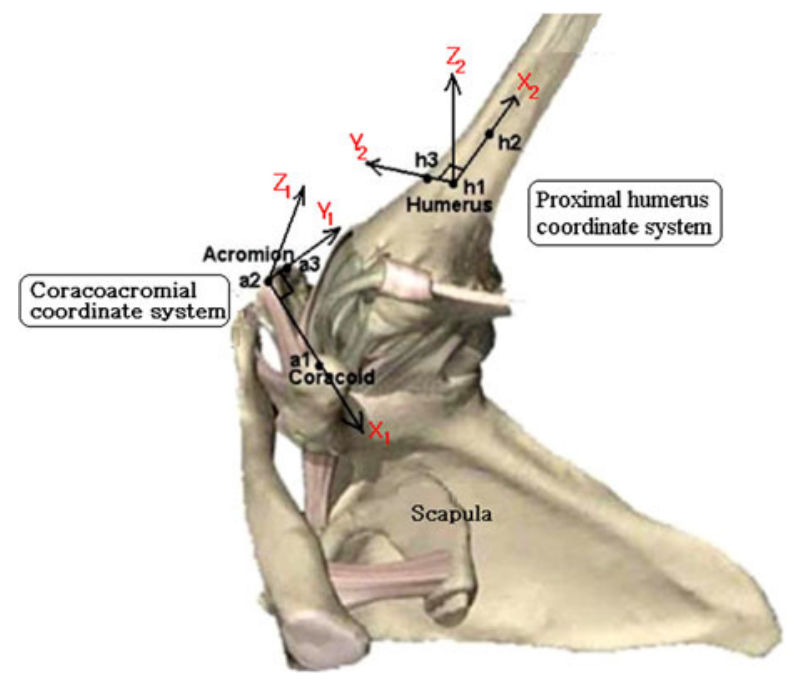

FIGURE 3. Schematic drawing showing the CACS and the PHCS.

extra-capsular CSs were completed, the glenohumeral joint capsule was removed, and the intra-capsular anatomical landmarks were digitized to define two intra-capsular CSs, i.e., the glenoid CS and the humeral head CS. Additionally, an extra-capsular humeral shaft CS was defined to find the direction of the humeral shaft axis (Fig. 3).

\section{In Situ Kinematic Data of Primary Extra-Capsular CSS}

For the in situ data collection, extra-capsular landmarks (three on the scapula and another three on the humerus) were assigned on the exterior surfaces of the coracoacromial complex and the proximal humerus so that they could be digitized without violating the glenohumeral capsule. With these anatomic landmarks, two extra-articular CSs were defined: CS1:CACS and CS2:PHCS (Fig. 3). Kinematic data for CACS and PHCS were then collected throughout the humeral rotational range of motion at $15^{\circ}$ increments.

\section{Coracoacromial Coordinate System}

The CACS was determined with three anatomic landmarks on the coracoacromial bony complex: the superolateral facet of the coracoid process $(a 1$ in Fig. 3), the anterolateral acromion and posterolateral acromion ( $a 2$ and $a 3$ in Fig. 3). The origin of the CACS was specified as the anterolateral acromion (a2) with the $X$-coordinate in the direction of the coracoid process. The $Y$-coordinate of the CACS was toward the posterolateral acromion ( $a 2)$ on the plane determined by three landmarks ( $a 1, a 2$, and $a 3$ ), and the $Z$-coordinate of the CACS was defined by the righthand rule-based determination of the Cartesian CS. 


\section{Proximal Humerus Coordinate System}

The PHCS was determined with two anatomic landmarks along the bicipital groove ( $h 1, h 2$ in Fig. 3) and a third anatomic landmark on the proximal humerus ( $h 3$ in Fig. 3). The third anatomic landmark was assigned toward the lateral direction to have more than $2 \mathrm{~cm}$ 'normal' distance from the line (h1 to h2) on the bicipital groove. The origin of the PHCS was located at the landmark $\mathrm{h} 1$ and the $X$-coordinate was along the bicipital groove ( $h 1$ to $h 2$ direction in Fig. 3). The $Y$-coordinate of the PHCS was toward the third landmark $(h 3)$ on the plane determined by the three landmarks $(h 1, h 2$, and $h 3)$, and the $Z$-coordinate of the PHCS was defined by the right-hand rule-based determination of the Cartesian CS.

\section{Intra-Capsular CSs and the Humeral Shaft CS}

Once the humeral external rotation data collection was completed, the joint capsule was removed and the anatomic data for the glenoid and the humerus were collected. Thereafter, two intra-capsular and one extracapsular CSs were defined: CS3:GCS, CS4:HHCS, and CS5:HSCS

\section{Glenoid Coordinate System}

The GCS was defined with points on the bony glenoid rim (Fig. 4). The determination of the GCS was executed in two stages. The preliminary CS was established on the SI axis defined by the intersection of the superior glenoid rim with the glenoid-coracoid confluence ( $S$ in Fig. 4), and the intersection of the inferior glenoid rim with the inferolateral margin of the scapula ( $I$ in Fig. 4). The initial location of the digitized points is inevitably observer-dependent; however, the SI and AP directions are determined mathematically so that the directions are reproducible. Anterior and posterior anatomic landmarks of the glenoid were defined as the anterior and posterior intersections ( $A$ and $P$ in Fig. 4) of the bony glenoid rim with a perpendicular plane bisecting the $S-I$ bony line. The bisecting point of the SI axis was assigned as the origin $\left(\vec{O}_{3}^{\prime}\right)$ of the preliminary glenoid coordinate system (preGCS). ${ }^{3}$ With $\vec{O}_{3}^{\prime}$, the anterior and posterior landmarks $(A, P)$ and the superior landmark $(S)$, the pre-GCS was determined by the right-hand rule-based Cartesian CS. The vector parallel to $\overrightarrow{\mathrm{AP}}$ and passing $\vec{O}_{3}^{\prime}$ was defined as the $X$-coordinate, whereas $\overrightarrow{\mathrm{SI}}$ was defined as the $Y$-coordinate of the pre-GCS. The $Z$-axis was then determined by the cross product of the $X$ and $Y$ axes.

The origin of the GCS was determined by translating the origin of the pre-GCS to the bottom of the glenoid surface, enabling the assessment of humeral kinematics with respect to the glenoid surface CS. This bottom level of the glenoid surface was determined by numerically finding the point $\left(\vec{g}_{h}\right)$ which has the least distance from the origin of the pre-GCS among the points $\left(\vec{g}_{i}\right)$ digitized along the concave SI glenoid surface. The vertical depth of the glenoid $(\Delta t)$ was expressed as the distance from the origin of the preGCS to the point $\left(\vec{g}_{h}\right)$ along the $Z$-direction of the pre-GCS Eq. (1) where $\overrightarrow{k_{3}}$ is the unit vector along the $Z$-axis of the pre-GCS.

$$
\Delta t=\left|\left(\vec{O}_{3}^{\prime}-\vec{g}_{h}\right) \cdot \vec{k}_{3}\right|
$$

\section{Humeral Head Coordinate System}

The HHCS was defined from digitized points along the articular margin of the humeral head where the

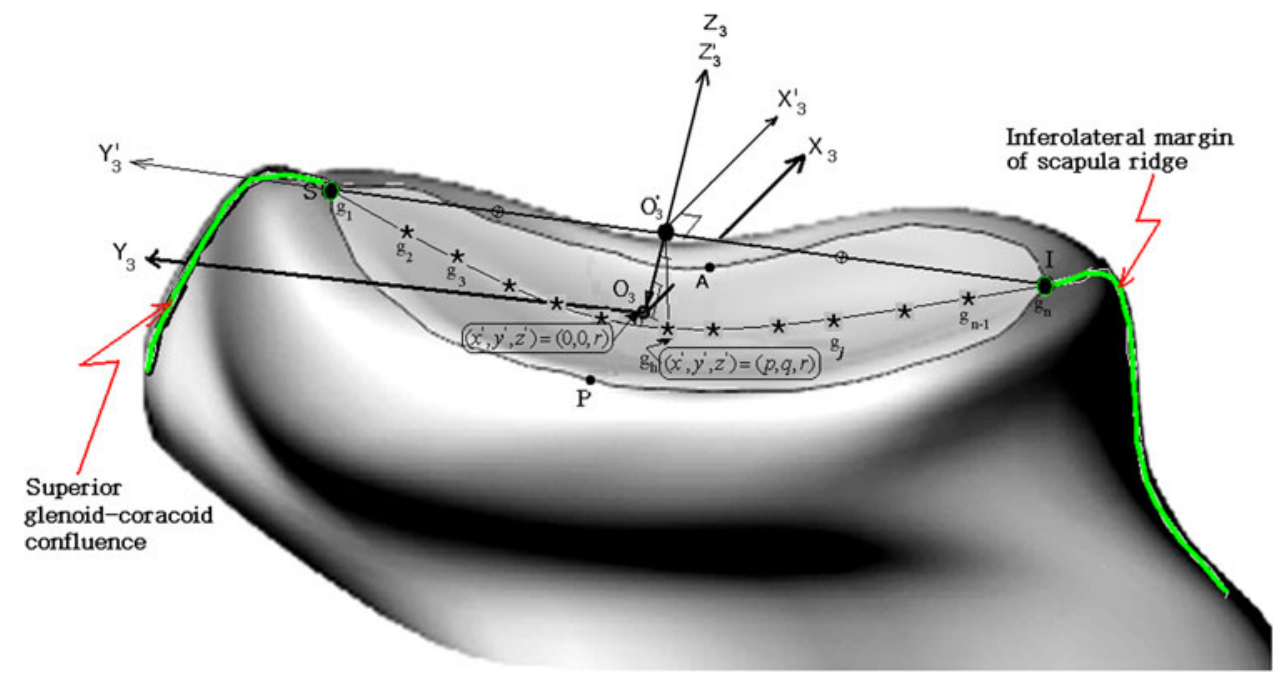

FIGURE 4. Glenoid coordinate system. 


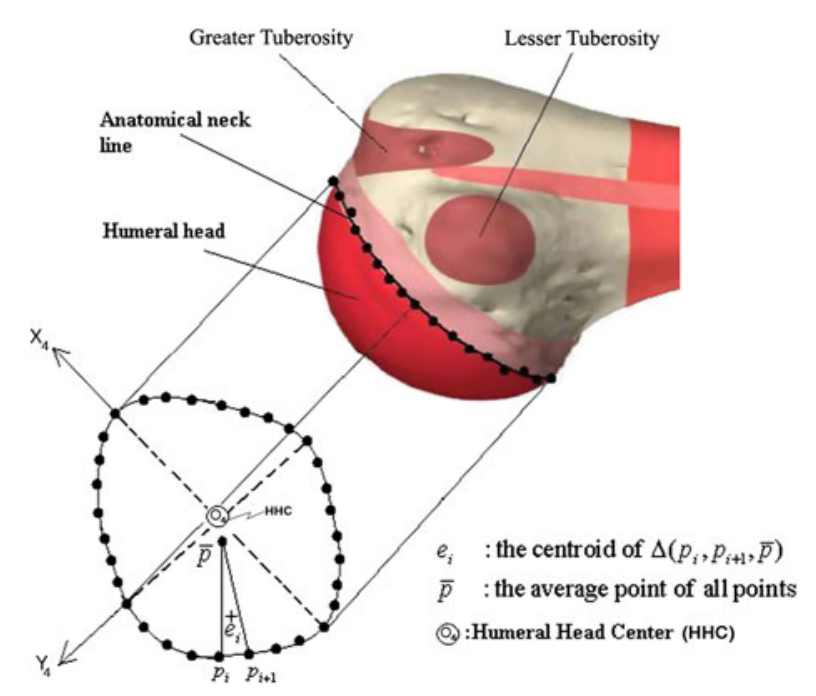

FIGURE 5. Humeral head coordinate system.

articular cartilage ends (Fig. 5). The origin $\left(\vec{O}_{4}\right)$ of the HHCS was defined as the true geometric centroid of the elliptical humeral head articular border. To determine the origin $\left(\vec{O}_{4}\right)$ of the HHCS, first, the average position $(\vec{p})$ of all the digitized humeral head articular margin points was calculated. The true centroid $\left(\vec{O}_{4}\right)$ and normal vector of the articular margin plane were then calculated by averaging the values of all triangles constructed from the average position $(\overrightarrow{\vec{p}})$, the $i$ th point $\left(\vec{p}_{i}\right)$, and $(i+1)$ th point $\left(\vec{p}_{i+1}\right)$. To numerically find the true origin of the HHCS, the area of each triangle was then calculated and the area fraction ratio $\left(s_{i}\right)$ defined as the ratio of the area $\left(Q_{i}\right)$ of a triangle $\left(\Delta\left(\overrightarrow{\vec{p}}, \vec{p}_{i}, \vec{p}_{i+1}\right)\right)$ to the total area of all triangles $(Q)$ was determined. The numerical expression of the area fraction ratio of a triangle relative to the total area is expressed as Eqs. (2)-(5), where $m$ is the number of digitized points.

$$
s_{i}=Q_{i} / Q
$$

where

$$
\begin{gathered}
Q=\sum_{i=1}^{m} Q_{i} \\
Q_{i}=\text { the area of the triangle } \Delta\left(\overrightarrow{\bar{p}}, \vec{p}_{i}, \vec{p}_{i}\right) \\
\overrightarrow{\bar{p}}=\frac{1}{m}\left(\sum_{i=1}^{m} \vec{p}_{i}\right)
\end{gathered}
$$

The centroid $\left(e_{i}\right)$ of each triangle was then calculated and the true origin $\left(\vec{O}_{4}\right)$ of the HHCS was defined as the average of the centroids of all triangles as shown in Eqs. (6) and (7).

$$
\begin{aligned}
\vec{O}_{4} & =\sum_{i=1}^{m}\left[\vec{e}_{i} \times s_{i}\right] \\
& =\vec{p}_{\mathrm{HHC}}
\end{aligned}
$$

where

$$
\vec{e}_{i}=\left(\overrightarrow{\vec{p}}+\vec{p}_{i}+\vec{p}_{i+1}\right) / 3
$$

The Z-coordinate of the HHCS was defined as the normal direction $(\overrightarrow{\vec{n}})$ of the humeral head resection plane and the normal vector $\left(\vec{n}_{i}\right)$ of each triangle was calculated by Eq. (8). Because the triangular planes are not parallel to each other, the normal direction of the HHCS was determined by averaging the normal directions of all triangles as Eq. (9).

$$
\vec{n}_{i}=\left(\vec{p}_{i}-\vec{p}_{\mathrm{HHC}}\right) \times\left(\vec{p}_{i+1}-\vec{p}_{\mathrm{HHC}}\right) /\left(\left|\vec{p}_{i}-\overrightarrow{\vec{p}}\right|\left|\vec{p}_{i+1}-\overrightarrow{\vec{p}}\right|\right)
$$

$$
\overrightarrow{\vec{n}}=\frac{1}{m}\left(\sum_{i=1}^{m} \overrightarrow{n_{i}}\right)
$$

The $X$-coordinate of the HHCS was assigned as the vector $\vec{O}_{4}$ and the superior point of the humeral head resection surface. The $Y$-coordinate of the HHCS was then calculated as the cross product of the $Z$-coordinate and $X$-coordinate of the HHCS.

\section{Humeral Shaft Coordinate System}

The HSCS was determined with two PVC cylinder circumferences formed by two sets of three points (Fig. 6). The two sets of points were positioned along the cylinder circumference $10 \mathrm{~cm}$ from each other. A circle was defined from the first set of three points and the center calculated to define the first center point $(c 1$ in Fig. 6). A circle was defined by the second set of three points on the PVC cylinder circumference and the center calculated to define the second center point ( $c 2$ in Fig. 6). The vector connecting the first and second center points was defined as the long axis of the proximal humerus, the Z-coordinate of the HSCS. The $X$-coordinate of the HSCS was determined as the vector connecting the first center point and one of the digitized points of the PVC circumference 1 . The $Y$-coordinate of the HSCS was determined by the cross product of the $Z$-coordinate and $X$-coordinate of the HSCS.

\section{In Situ Kinematic Data of Intra-Capsular CSS}

Spatial relationships among CSs were then obtained. Using the constant spatial relationship, kinematic data of the humeral head center (HHC), the 


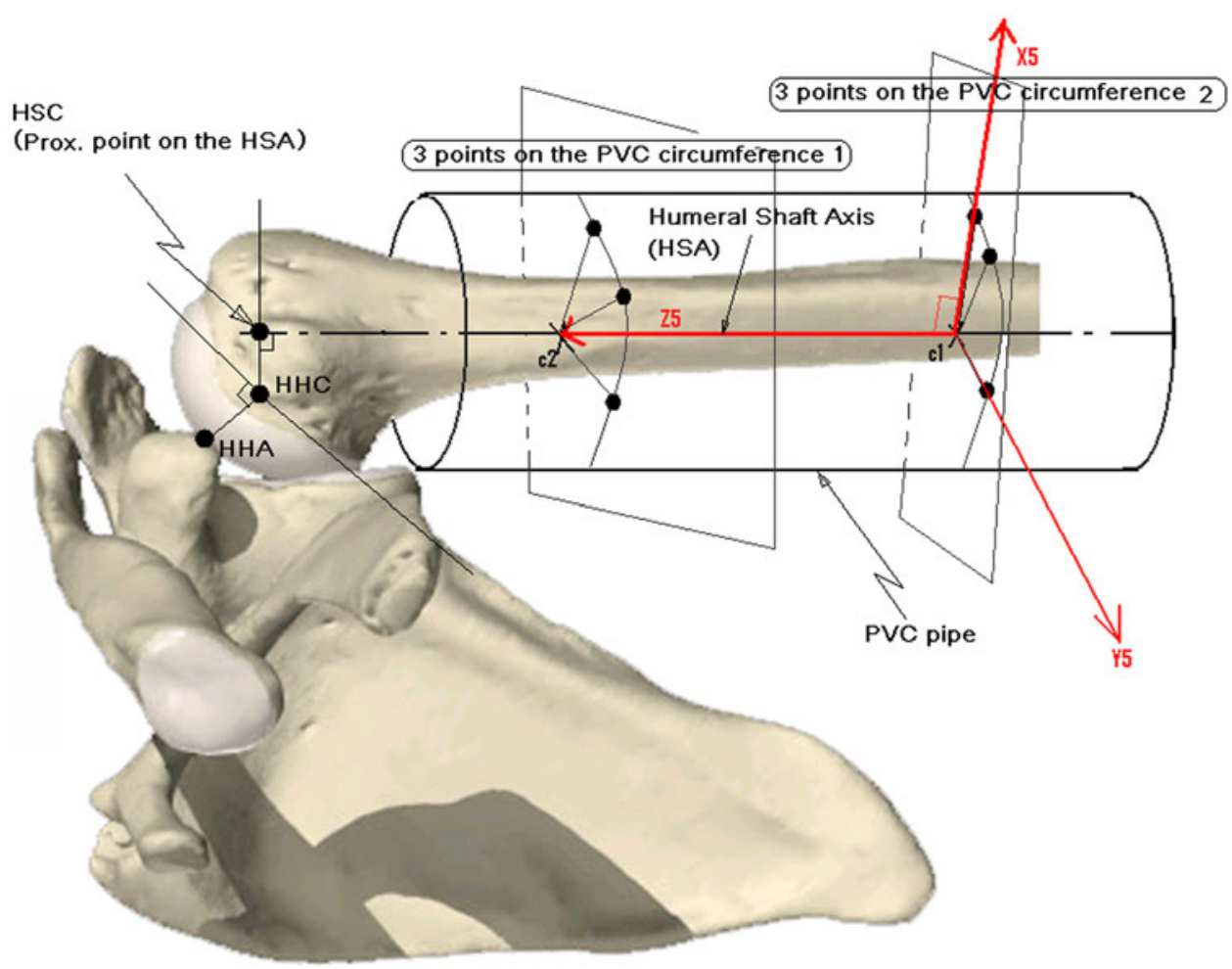

FIGURE 6. Humeral shaft coordinate system.

humeral head apex (HHA), and the humeral shaft center (HSC) were calculated with respect to humeral rotation positions.

\section{Humeral Points of Interest: $H H C, H H A, H S C$}

Kinematic data of the HHC, HHA, and HSC were quantified at each humeral rotation position. The HHC was defined as the origin $\left(\vec{O}_{4}\right)$ of the HHCS. The HSC was defined by intersecting the humeral shaft axis with a plane normal to the humeral shaft axis at the level of the HHC (Fig. 6). To determine HHA the points along the articular surface were digitized and the point $\left(\alpha_{f}\right)$ furthest away from the origin of the HHCS was found $\left(\alpha_{i}\right)$. The HHC was then projected onto the proximal humeral head articular surface along the $Z$-coordinate by the height of the furthest point $(\Delta h)$ (Fig. 7). The mathematical expression of $\Delta h$ is Eq. (10).

$$
\Delta h=\max \left[\left(\vec{O}_{4}-\vec{\alpha}_{i}\right) \cdot \vec{k}_{4}\right]
$$

\section{Quantified Parameters}

Quantified parameters included anatomical and geometric characteristics of the glenoid, i.e., AP and SI

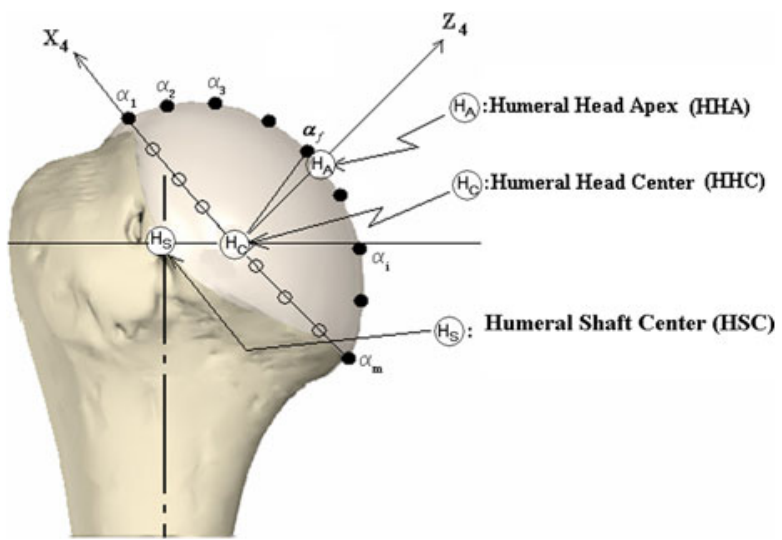

FIGURE 7. Position of anatomical parameters of the humerus.

lengths and depths of the glenoid. In addition, humeral rotational range of motion, glenohumeral translation, and the path of the HHA, HHC, and HSC with respect to the glenoid were also determined.

\section{Accuracy, Repeatability, and Reliability}

To assess reliability of the proposed landmark determination methods, accuracy, and repeatability for measuring the $\mathrm{HHC}$, and intra- and inter-observer reliability for the origin of the GCS, HHC and HHA 
were calculated. The accuracy of measuring the HHC was measured by checking the geometric center of a sphere based on a local CS defined along the equator of the sphere. Since the location of the HHC may vary depending on the level of digitization with respect to the equator, the $X, Y$ positions of the center of the sphere were compared to five trials of digitizing the equator of the sphere to simulate humeral head articulation digitization. The intra-observer and interobserver reliability were determined for the origin of the GCS, HHC, and HHA by digitizing a specimen and averaging the repeatability of five trials in three dimensions. For the inter-observer reliability test, two persons participated.

\section{Statistical Analysis}

The repeatability for determination of the $\mathrm{HHC}$ was calculated as the standard deviation of data measured by an observer. Intra- and inter-observer reliabilities were calculated with standard deviation between interand intra-observer data sets. Additionally, correlation analyses were performed using multiple linear regression to assess interdependencies between anatomical parameters (AP \& SI lengths or AP \&SI depths) of the glenoid and the humeral translations in the AP, SI, and ML directions when rotated from maximum internal to maximum external rotation. A $p$-value of 0.05 was used to determine significance.

\section{RESULTS}

\section{Accuracy and Repeatability of the Landmark Determination}

The accuracy and repeatability in calculation of the $\mathrm{HHC}$ were determined to be 0.44 and $0.41 \mathrm{~mm}$, respectively. The intra-observer reliability for determining the origin of the GCS, HHC, and HHA was within $0.26,0.29$, and $0.42 \mathrm{~mm}$, respectively. The interobserver reliability for the origin of the GCS, HHC, and HHA was within $0.37,0.30$, and $1.75 \mathrm{~mm}$, respectively.

\section{Glenohumeral Joint Kinematics}

The maximum internal and external rotations were $-14.2 \pm 14.1^{\circ}$ and $145.0 \pm 16.6^{\circ}$, respectively. As the humerus rotated from maximum internal to maximum external rotation, the HHA and HHC moved anteroinferior to anterosuperior (Fig. 8). On the contrary, the HSC moved posterosuperior to posteroinferior from maximum internal to maximum external rotation. At $45^{\circ}$ and $60^{\circ}$ of external rotation, the locations of the HHC and HHA were very close to the glenoid center on the AP-SI plane of the GCS (Fig. 8).

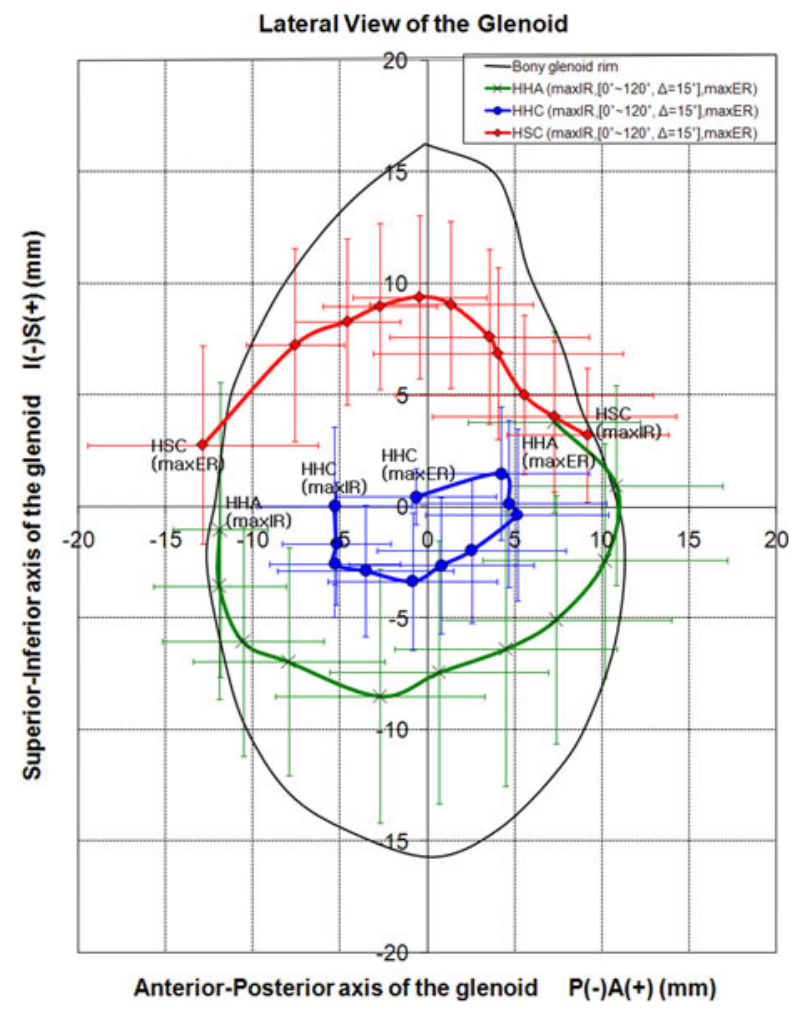

FIGURE 8. Paths of the HHA, the HHC, the HSC on the lateral view of the glenoid. The standard deviations for AP and SI coordinates are not shown here.

In the medial-lateral direction, the locations of the HHA and $\mathrm{HHC}$ were closest to the glenoid center at $45^{\circ}$ and $60^{\circ}$ of external rotation (Fig. 9). At maximum external rotation, the locations of the HHA and HHC were furthest away (lateral direction) from the glenoid center (laterally 9.0 and $18.8 \mathrm{~mm}$ for the HHA and HHC, respectively). The location of the HSC was 19.8 and $18.5 \mathrm{~mm}$ lateral in the maximum internal rotation and maximum external rotation positions, respectively. In the medial-lateral direction, the paths of the HHA and $\mathrm{HHC}$ were different from that of the HSC during external humeral rotation. The HHA and the HHC moved medial toward the glenoid surface 5.3 and $4.2 \mathrm{~mm}$, respectively, from maximum internal rotation to $45^{\circ}$ to $60^{\circ}$ of external rotation, and then returned (Fig. 9). On the contrary, the HSC moved slightly lateral, and then returned medial from maximum internal to maximum external rotation.

Maximum translations of each anatomic landmark of the humerus were calculated as the difference between the maximum and the minimum values throughout the full range of humeral rotation (Table 1). For both the AP and SI directions, the maximum translation of the $\mathrm{HHC}$ was smaller than that of the HHA or HSC $(p=0.04)$. In the mediallateral direction, the translation of the $\mathrm{HSC}$ was smaller than that of the HHC and HHA. 


\section{Correlation Between Anatomical Dimensions and Humeral Translations}

There was a significant correlation between the AP length of the glenoid and the maximum AP translation of the HHC $(R=0.9, p=0.014)$. The ratio of the smaller AP depth with respect to the SI depth of the

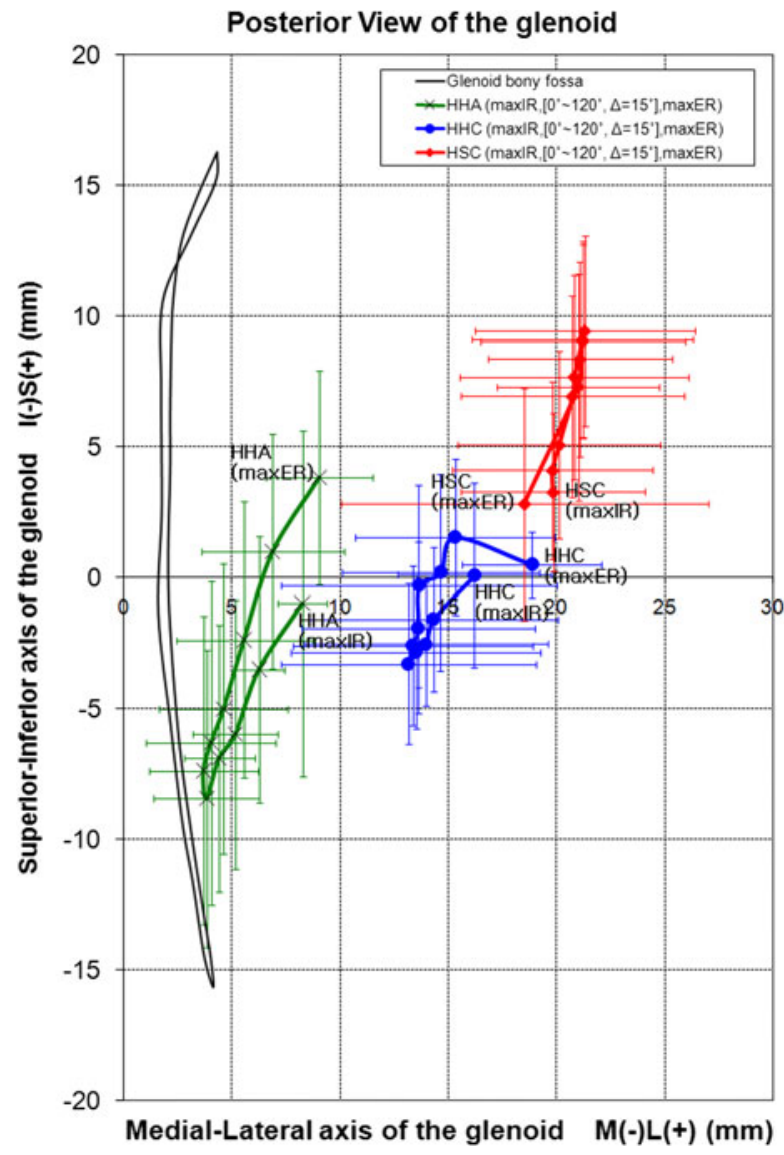

FIGURE 9. Paths of the HHA, the HHC, and the HSC on the posterior view of the glenoid. The standard deviations for AP and SI coordinates are not shown. glenoid $(27.3 \pm 16.5 \%)$ was significantly correlated to the ratio of the maximum AP/SI HHA translation ratio $(191.4 \pm 43.8 \%, R=0.896, p=0.02)$, while not significantly for $\mathrm{HHC}(55.0 \pm 21.9 \%, R=0.324$, $p=0.53)$ and for HSC $(184.0 \pm 118.4 \% R=0.442$, $p=0.38)$.

\section{DISCUSSION}

This study provides a robust method for quantifying detailed anatomically relevant and specimen-specific glenohumeral joint kinematics. The current ISB recommendation, while highly effective for studying in vivo shoulder kinematics, does not provide an anatomically detailed protocol for defining a glenoid CS.

One of the differences between the ISB recommendation and the proposed method is that only the middle and the proximal humerus is required for the current method. The ISB recommendation defines the humeral CS using the glenohumeral rotation center, medial and lateral epicondyles. The glenohumeral rotation center is estimated by regression of continuous instantaneous intersections of the humeral shaft axis. In contrast, in our study, the humeral CS is with the anatomical $\mathrm{HHC}$ and the axis of the proximal humeral diaphysis.

The external rotation and abduction of the humerus as defined by the ISB recommendation are based on the sum of the scapulothoracic and glenohumeral kinematics. ${ }^{6,14,25}$ In cadaveric studies glenohumeral kinematics are commonly investigated due to its large rotational movement relative to that of the scapulothorax. This study provides a glenohumeral kinematic protocol that enables the assessment of asymmetric glenohumeral kinematic features, since anatomic landmarks, anatomical directions, and CSs are determined by precise and reproducible methods.

TABLE 1. Anatomic dimensions of the glenoid and the maximum humeral translation in each direction when rotating the humerus from maximum internal to maximum external axial rotation.

\begin{tabular}{|c|c|c|c|c|c|c|c|c|c|c|c|c|c|}
\hline \multirow[b]{2}{*}{ Spec\# } & \multicolumn{4}{|c|}{ Glenoid dimension $(\mathrm{mm})$} & \multicolumn{3}{|c|}{$\begin{array}{l}\text { Maximum translation } \\
\text { of HHA }(\mathrm{mm})\end{array}$} & \multicolumn{3}{|c|}{$\begin{array}{c}\text { Maximum translation } \\
\text { of } \mathrm{HHC}(\mathrm{mm})\end{array}$} & \multicolumn{3}{|c|}{$\begin{array}{l}\text { Maximum translation } \\
\text { of } \mathrm{HSC}(\mathrm{mm})\end{array}$} \\
\hline & AP length & SI length & AP depth & SI depth & AP & SI & ML & AP & SI & $\mathrm{ML}$ & AP & SI & $\mathrm{ML}$ \\
\hline 1 & 23.0 & 29.3 & 0.8 & 3.1 & 31.7 & 17.1 & 4.5 & 16.6 & 9.6 & 2.8 & 25.0 & 8.3 & 0.8 \\
\hline 2 & 20.6 & 28.0 & 0.1 & 3.8 & 18.1 & 11.8 & 8.7 & 12.3 & 6.2 & 4.5 & 9.2 & 7.7 & 5.6 \\
\hline 3 & 19.9 & 28.3 & 1.5 & 3.6 & 27.3 & 11.9 & 9.0 & 11.1 & 4.9 & 4.7 & 26.1 & 13.8 & 7.8 \\
\hline 4 & 17.3 & 26.1 & 0.5 & 2.8 & 23.3 & 17.6 & 5.1 & 7.9 & 7.3 & 2.3 & 30.2 & 12.8 & 4.2 \\
\hline 5 & 18.3 & 28.3 & 0.6 & 2.2 & 24.1 & 12.0 & 6.0 & 10.7 & 3.9 & 2.6 & 33.0 & 8.7 & 1.9 \\
\hline 6 & 23.9 & 32.0 & 2.0 & 4.1 & 26.2 & 10.6 & 8.0 & 26.2 & 10.6 & 20.4 & 18.2 & 13.5 & 5.4 \\
\hline Avg. & 20.5 & 28.7 & 0.9 & 3.3 & 25.1 & 13.5 & 6.9 & 14.1 & 7.1 & 6.2 & 23.6 & 10.8 & 4.3 \\
\hline STD & 2.6 & 2.0 & 0.7 & 0.7 & 4.5 & 3.0 & 1.9 & 6.6 & 2.6 & 7.0 & 8.7 & 2.8 & 2.6 \\
\hline
\end{tabular}

Note: Spec\#, Avg., and STD represents specimen number, average, and standard deviation, respectively. AP, anterior(+)/posterior(-); $\mathrm{SI}$, superior(+)/inferior(-); $L M$, lateral(+)/medial(-). 
The proposed anatomically based protocol provides a specimen-specific method for quantifying asymmetric kinematics of the glenohumeral joint. Previous glenohumeral joint kinematic studies have used the assumption of a sphere in representing the humeral head or both the humeral head and glenoid ${ }^{5,16}$ which was later contradicted by Iannotti et al. ${ }^{9}$ In particular, Iannotti et al. reported that the ratio of the SI lengthto-AP length of the glenoid was 1.3, and the SI depth of the glenoid was 2.6 times the AP depth, so the humeral head is oval rather than spherical. ${ }^{9}$ In this study, the ratio of the smaller AP depth with respect to the SI depth of the glenoid was significantly correlated to the ratio of the maximum AP/SI HHA translation ratio. The current protocol maps the anatomic landmarks of the humerus to the glenoid CS. During axial rotation of the humerus, the $\mathrm{HHC}$ moved the least in any direction. ${ }^{22}{ }^{23}$ Buchler et al. $^{4}$ also reported similarily that the glenohumeral contact region remained near the center of the glenoid fossa for normal shoulders.

To minimize irreproducibility arising from discrepancy in initial specimen position and inter-observer or intra-observer error in determining CSs, several protocols for reproducible positioning were employed. Since the locations of digitized points are somewhat user-dependent, mathematical treatments are required to reproducibly determine anatomical landmarks among different observers or trials. Among the mathematical treatments proposed in the current glenohumeral kinematic protocol, the most important ones are related to the glenoid and humerus: the bottom of the glenoid as the projection of the origin of the pre-GCS to the SI line of the glenoid surface, the HSC line as the line connecting mathematically determined centers of two perimeters of the PVC pipe, and the $\mathrm{HHC}$ as the center of mass of the humeral neck plane.

This study has limitations which may influence the calculations of glenohumeral kinematics. The glenohumeral joint was left intact but muscle loading was not used. Instead, glenohumeral joint compression of $22 \mathrm{~N}$ was used to stabilize the joint during articulation. ${ }^{21}$ If muscle loading was applied the glenohumeral translation with humeral rotation would have been less. Another potential limitation of the application of this method in this particular study was the lack of scapulohumeral motion. However, since both the scapula and humerus CSs are defined this method could be used in the presence of scapulohumeral motion. While the extracapsular landmarks are somewhat still conceptual they do not affect the output of the anatomically based glenohumeral kinematics. The coracoacromial CS and the proximal humerus CS are used as initial reference CS for the secondary CSs that are defined after capsular resection and geometry digitization. The spatial relationships between the primary reference CSs and intracapsular CSs, or the humeral shaft CS are assessed by rigid bone assumption. The precise location of the primary CSs is not crucial to the data output, which is defined solely by the secondary CSs.

This study defines reproducible CSs for glenohumeral kinematic studies based on bony landmarks rather than on musculoskeletal kinematic data. In addition, it should be recognized that all five CSs are not necessary to be used at all times and that the extracapsular CSs are used to determine the intra-capsular landmark kinematics without violating the capsule. Each investigator can choose to use pertinent coordinates for a specific pathology to be studied. This study provides a repeatable glenohumeral kinematic protocol that enables the assessment of asymmetric glenohumeral kinematics, since anatomic landmarks, anatomical directions, and CSs are determined by precise and reproducible methods.

\section{ACKNOWLEDGMENTS}

This study was supported by VA Rehab R\&D, VA Medical Research and John C. Griswold Foundation. The sponsors had no role in the study design, collection, analysis, or interpretation of data, writing of the manuscript, or in the decision to submit the paper for publication.

\section{OPEN ACCESS}

This article is distributed under the terms of the Creative Commons Attribution Noncommercial License which permits any noncommercial use, distribution, and reproduction in any medium, provided the original author(s) and source are credited.

\section{REFERENCES}

\footnotetext{
${ }^{1}$ Baeyens, J. P., P. Van Roy, and J. P. Clarys. Intra-articular kinematics of the normal glenohumeral joint in the late preparatory phase of throwing: Kaltenborn's rule revisited. Ergonomics 43:1726-1737, 2000.

${ }^{2}$ Baeyens, J. P., P. Van Roy, A. De Schepper, G. Declercq, and J. P. Clarijs. Glenohumeral joint kinematics related to minor anterior instability of the shoulder at the end of the late preparatory phase of throwing. Clin. Biomech. (Bristol, Avon) 16:752-757, 2001.

${ }^{3}$ Beaulieu, C. F., D. K. Hodge, A. G. Bergman, K. Butts, B. L. Daniel, C. L. Napper, R. D. Darrow, C. L. Dumoulin, and R. J. Herfkens. Glenohumeral relationships during physiologic shoulder motion and stress testing: initial experience with open MR imaging and active imaging-plane registration. Radiology 212:699-705, 1999.
} 
${ }^{4}$ Buchler, P., N. A. Ramaniraka, L. R. Rakotomanana, J. P. Iannotti, and A. Farron. A finite element model of the shoulder: application to the comparison of normal and osteoarthritic joints. Clin. Biomech. (Bristol, Avon) 17:630-639, 2002.

${ }^{5}$ Engin, A. E., and S. T. Tumer. Three-dimensional kinematic modelling of the human shoulder complex - Part I: physical model and determination of joint sinus cones. J. Biomech. Eng. 111:107-112, 1989.

${ }^{6}$ Fleisig, G. S., J. R. Andrews, C. J. Dillman, and R. F. Escamilla. Kinetics of baseball pitching with implications about injury mechanisms. Am. J. Sports Med. 23:233-239, 1995.

${ }^{7}$ Gupta, R., and T. Q. Lee. Positional-dependent changes in glenohumeral joint contact pressure and force: possible biomechanical etiology of posterior glenoid wear. J. Shoulder Elbow Surg. 14:105S-110S, 2005.

${ }^{8}$ Huffman, G. R., J. E. Tibone, M. H. McGarry, B. M. Phipps, Y. S. Lee, and T. Q. Lee. Path of glenohumeral articulation throughout the rotational range of motion in a thrower's shoulder model. Am. J. Sports Med. 34:16621669, 2006.

${ }^{9}$ Iannotti, J. P., J. P. Gabriel, S. L. Schneck, B. G. Evans, and S. Misra. The normal glenohumeral relationships. An anatomical study of one hundred and forty shoulders. J. Bone Joint Surg. Am. 74:491-500, 1992.

${ }^{10}$ Lee, T. Q., A. D. Black, J. E. Tibone, and P. J. McMahon. Release of the coracoacromial ligament can lead to glenohumeral laxity: a biomechanical study. J. Shoulder Elbow Surg. 10:68-72, 2001.

${ }^{11}$ McMahon, P. J., S. Chow, L. Sciaroni, B. Y. Yang, and T. Q. Lee. A novel cadaveric model for anterior-inferior shoulder dislocation using forcible apprehension positioning. J. Rehabil. Res. Dev. 40:349-359, 2003.

${ }^{12}$ McMahon, P. J., D. T. Dee, B. Y. Yang, and T. Q. Lee. Mal-aligning humeral offset may not effect shoulder hemiarthroplasty: a biomechanical study. Med. Sci. Monit. 9:CR346-CR352, 2003.

${ }^{13}$ Mihata, T., Y. Lee, M. H. McGarry, M. Abe, and T. Q. Lee. Excessive humeral external rotation results in increased shoulder laxity. Am. J. Sports Med. 32:12781285, 2004.

${ }^{14}$ Miyashita, K., H. Kobayashi, S. Koshida, and Y. Urabe. Glenohumeral, scapular, and thoracic angles at maximum shoulder external rotation in throwing. Am. J. Sports Med. 38(2):363-368, 2009.

${ }^{15}$ Nagerl, H., D. Kubein-Meesenburg, H. Cotta, J. Fanghanel, and S. Kirsch. Biomechanical principles in diarthroses and synarthroses. II: the humerus articulation as a ball-andsocket joint. Z. Orthop. Ihre. Grenzgeb. 131:293-301, 1993.

${ }^{16}$ Novotny, J. E., B. D. Beynnon, and C. E. Nichols, 3rd. A numerical solution to calculate internal-external rotation at the glenohumeral joint. Clin. Biomech. (Bristol, Avon) 16:395-400, 2001.

${ }^{17}$ Panossian, V. R., T. Mihata, J. E. Tibone, M. J. Fitzpatrick, M. H. McGarry, and T. Q. Lee. Biomechanical analysis of isolated type II SLAP lesions and repair. J. Shoulder Elbow Surg. 14:529-534, 2005.

${ }^{18}$ Sciaroni, L. N., P. J. McMahon, T. G. Cheung, and T. Q. Lee. Open surgical repair restores joint forces that resist glenohumeral dislocation. Clin. Orthop. Relat. Res. 400: 58-64, 2002.

${ }^{19}$ Shafer, B. L., T. Mihata, M. H. McGarry, J. E. Tibone, and T. Q. Lee. Effects of capsular plication and rotator interval closure in simulated multidirectional shoulder instability. J. Bone Joint Surg. Am. 90:136-144, 2008.

${ }^{20}$ Shapiro, T. A., M. H. McGarry, R. Gupta, Y. S. Lee, and T. Q. Lee. Biomechanical effects of glenoid retroversion in total shoulder arthroplasty. J. Shoulder Elbow Surg. 16(3 suppl):s90-s95, 2006.

${ }^{21}$ Tibone, J. E., P. J. McMahon, T. A. Shrader, M. D. Sandusky, and T. Q. Lee. Glenohumeral joint translation after arthroscopic, nonablative, thermal capsuloplasty with a laser. Am. J. Sports Med. 26:495-498, 1998.

${ }^{22}$ Veeger, H. E. The position of the rotation center of the glenohumeral joint. J. Biomech. 33:1711-1715, 2000.

${ }^{23}$ Woltring, H. J. Estimation of the trajectory of the instantaneous centre of rotation in planar biokinematics. J. Biomech. 23:1273-1274, 1990.

${ }^{24}$ Wu, G., F. C. van der Helm, H. E. Veeger, M. Makhsous, P. Van Roy, C. Anglin, J. Nagels, A. R. Karduna, K. McQuade, X. Wang, F. W. Werner, and B. Buchholz. ISB recommendation on definitions of joint coordinate systems of various joints for the reporting of human joint motion-Part II: shoulder, elbow, wrist and hand. J. Biomech. 38:981-992, 2005.

${ }^{25}$ Yano, Y., J. Hamada, K. Tamai, K. Yoshizaki, R. Sahara, T. Fujiwara, and Y. Nohara. Different scapular kinematics in healthy subjects during arm elevation and lowering: glenohumeral and scapulothoracic patterns. J. Shoulder Elbow Surg. 19(2):209-215, 2009.

${ }^{26}$ Youm, T., N. S. ElAttrache, J. E. Tibone, M. H. McGarry, and T. Q. Lee. The effect of the long head of the biceps on glenohumeral kinematics. J. Shoulder Elbow Surg. 18:122129, 2009

${ }^{27}$ Youm, T., J. E. Tibone, N. S. ElAttrache, M. H. McGarry, and T. Q. Lee. Simulated type II superior labral anterior posterior lesions do not alter the path of glenohumeral articulation: a cadaveric biomechanical study. Am. J. Sports Med. 36:767-774, 2008.

${ }^{28}$ Yu, J., M. H. McGarry, Y. S. Lee, L. V. Duong, and T. Q. Lee. Biomechanical effects of supraspinatus repair on the glenohumeral joint. J. Shoulder Elbow Surg. 14:65S-71S, 2005. 